\title{
Elevated IL-1 $\alpha$ and CXCL10 serum levels occur in patients with homozygous sickle cell disease and a history of acute splenic sequestration
}

\author{
Adel Driss ${ }^{\mathrm{a}, *}$, Nana O. Wilson ${ }^{\mathrm{a}}$, Karlene Mason ${ }^{\mathrm{b}}$, Hyacinth I. Hyacinth ${ }^{\mathrm{a}}$, Jacqueline M. Hibbert ${ }^{\mathrm{a}}$, \\ Graham R. Serjeant ${ }^{\mathrm{b}}$ and Jonathan K. Stiles ${ }^{\mathrm{a}}$ \\ ${ }^{a}$ Department of Microbiology, Biochemistry and Immunology, Morehouse School of Medicine, Atlanta, GA, USA \\ ${ }^{\mathrm{b}}$ Sickle Cell Trust, Kingston, Jamaica
}

\begin{abstract}
Acute splenic sequestration (ASS) and chronic hypersplenism are common features of homozygous sickle cell (SS) disease in the first 5 years of life affecting one-third of subjects in the Jamaican Cohort Study. The risk factors are largely unknown and the current study explores a possible role of genetic factors. We have explored these in subjects who received splenectomy in the management of ASS $(n=8)$ or chronic hypersplenism $(n=9)$ along with age, gender, and genotype matched controls using Luminex Technology to assess 42 human cytokines/chemokines, including IL-1 $\alpha$ and CXCL10 (IP-10). Levels of IL-1 $\alpha$ $(p=0.008)$ and CXCL10 $(p=0.009)$ were significantly elevated in patients treated by splenectomy compared with the control group. Levels of IL- $1 \alpha$ were significantly higher in those with a history of ASS compared with matched normal controls ( $p=$ $0.028)$ but not in those treated for hypersplenism $(p=0.093)$. Furthermore, several significant differences were found in the median ratios of some cytokine biomarkers between the splenectomized group and the normal controls. These observations are consistent with acute splenic sequestration having a distinct phenotype which may be helpful in predicting those at risk of this complication and suggest that the mechanism of these differences merit further study.
\end{abstract}

Keywords: Sickle cell anemia, biomarkers, splenectomy, spleen, interleukins, cytokine, acute splenic sequestration, chronic hypersplenism

\section{Introduction}

Homozygous sickle cell (SS) disease, one of the most common genetic diseases in the world, is associated with marked variability in clinical and hematological features. Although high levels of fetal hemoglobin protect against sickling early in life, serious complications rapidly develop and the highest mortality occurs at 6-

\footnotetext{
* Corresponding author: Adel Driss, Department of Microbiology, Biochemistry and Immunology, Morehouse School of Medicine, 720 Westview Drive SW, Hugh Gloster Bldg., Suite 352, Atlanta GA 30310-1495, USA. Tel.: +1 404751 7850; Fax: +1 404752 1179; E-mail: adel@weboris.com; adriss@msm.edu.
}

12 months of age. Foremost among these causes of death are pneumococcal infections and acute splenic sequestration (ASS). The former has been effectively addressed by pneumococcal prophylaxis [5] and the latter by parental education in the early diagnosis of ASS [4]. At later ages, hypersplenism emerges as an important complication and differs from ASS with sustained marked splenomegaly and a new hematological equilibrium with lower hemoglobin and higher reticulocyte levels consistent with sustained rapid hemolysis and red cell survival which may be as short as one to three days. The natural history of these complications suggests a median age of one to three years for ASS and three to eight years for chronic hypersplenism. 
These two splenic complications appear to follow separate courses although hypersplenism may follow an attack of ASS and sometimes acute sequestration may be superimposed upon hypersplenism. Acute splenic sequestration may be more common in subjects with low fetal hemoglobin (HbF) levels [15] and possibly less common with alpha thalassemia [4] which inhibits sickling and although seasonal pattern or clustering of events has not been shown, concurrence of ASS attacks in identical twins [17] may support an environmental risk factor. Hypersplenism appears more common with high levels of $\mathrm{HbF}$ and alpha thalassemia, both inhibiting sickling and allowing splenomegaly to persist, and is commonly reported in SS patients in Eastern Province of Saudi Arabia [11] and central India [8], populations associated with these hematologic factors. In the absence of a clear pattern of hematological or environmental risk factors, the current study seeks potential genetic factors by measuring biomarkers among cytokines, chemokines, and growth factors in the hope of identifying risk factors useful in predicting the clinical course.

\section{Material and methods}

\subsection{Study subjects}

The patients attended the Sickle Cell Clinic at the University of the West Indies, Kingston, Jamaica which was operated until September 1999 by staff of the British Medical Research Council. Between June 1973 and Dec 1981, the Jamaican Cohort Study was established by screening 100,000 consecutive non-operative deliveries at the main government maternity hospital (Victoria Jubilee) with the detection of 311 babies with SS disease who have been followed from birth [13] with documentation of all clinical features. During this follow-up 37 subjects with SS disease received splenectomy prophylactically for ASS $(n=15)$ or therapeutically for hypersplenism $(n=22)$. Of the 15 subjects in the ASS group, 7 were not available (4 deaths, 3 emigrations), and the remaining 8 subjects participated whereas of the 22 hypersplenics, 13 were not available (5 deaths, 8 emigrations) and the remaining 9 all took part in this study which was performed in September and October 2010. The 8 patients in the ASS group (aged 29.8-30.9 years), were each matched by age and gender to $8 \mathrm{SS}$ controls (aged 28.8-31.1 years), closest in date of birth to the index case but without a history of ASS. The 9 patients in the hypersplenic group (aged
30.6-36.7 years), were similarly matched to 9 SS controls (aged 30.5-36.7 years). The final study therefore comprised 34 subjects, 17 with splenectomy (8 ASS, 9 hypersplenism) and 17 SS controls. Splenectomy in the hypersplenic group was performed at a median age of 4.8 years with a median follow-up of 28.6 years and the ASS group at 1.7 years with median follow-up of 27.5 years. All patients gave informed consent and the study was approved by the Institutional Review Board (IRB) of Morehouse School of Medicine (MSM) and the University of the West Indies.

\subsection{Clinical definitions}

Hypersplenism was defined as a spleen of $4 \mathrm{~cm}$ or more below the costal margin, $\mathrm{Hb}<6.5 \mathrm{~g} / \mathrm{dl}$, reticulocytes $>15 \%$ and platelets below $200 \times 10^{9} / 1$, observed on at least two occasions six months apart. Following splenectomy, all cases demonstrated an increase in hemoglobin and reduction in reticulocyte counts consistent with the diagnosis of hypersplenism. Acute splenic sequestration was defined as a sudden moderate increase in spleen size, usually $3-4 \mathrm{~cm}$ below the left costal margin, a fall in hemoglobin usually greater than $2 \mathrm{~g} / \mathrm{dl}$ and evidence of continued bone marrow activity (elevated reticulocyte or normoblast count) with resolution of these features following transfusion.

\subsection{Sample}

Ten $\mathrm{ml}$ of venous blood from the antecubital vein was taken into the P100 Blood Collection System ${ }^{\circledR}$ (Becton-Dickenson, Franklin Lakes, NJ), which contained protease inhibitors to preserve plasma proteins. After centrifugation at $1600 \mathrm{~g}$ for 30 minutes in a swing bucket centrifuge, the samples were aliquoted into cryovials, frozen at $-20^{\circ} \mathrm{C}$ and shipped on dry ice from Jamaica to the laboratory at Morehouse School of Medicine in Atlanta GA.

\subsection{Multiplexed microsphere cytokine immunoassay}

Plasma samples were evaluated for serological marker levels using the Milliplex (Luminex ${ }^{\circledR}$ Technology, Millipore, Billerica, MA) panel of 42 human Cytokines/Chemokines (Millipore): EGF, Eotaxin, FGF2, Flt-3 ligand, Fractalkine, G-CSF, GM-CSF, GRO, IFN- $\alpha 2$, IFN- $\gamma$, IL-10, IL-12 (p40), IL-12 (p70), IL-13, IL-15, IL-17, IL-1R $\alpha$, IL-1 $\alpha$, IL-1 $\beta$, IL-2, IL-3, IL-4, IL-5, IL-6, IL-7, IL-8, IL-9, CXCL10, MCP-1, MCP-3, MDC (CCL22), MIP-1 $\alpha$, MIP-1 $\beta$, PDGF-AA, PDGF- 
AB/BB, RANTES, TGF- $\alpha$, TNF- $\alpha$, TNF- $\beta$, VEGF, SCD40L, sIL-2R $\alpha$. These markers target a wide range of mechanisms involved in angiogenesis and inflammatory pathways. The multiplex bead-based cytokine assay is coupled with the Luminex system measuring biological factors with optimal concentrations of standards and antibodies. Briefly, duplicate plasma samples were diluted 1:4 in Bio-Plex sample diluents and an eight point, broad range cytokine standard was prepared by serial dilution from a cytokine stock. Fifty microliters of diluted sample or standard were transferred to a 96-well filtration plate containing antibody conjugated beads. The plate was agitated for $30 \mathrm{sec}-$ onds at $1,100 \mathrm{rpm}$, reduced to $300 \mathrm{rpm}$, and incubated for 60 minutes at room temperature. After incubation and several washes, detection antibody $(2 \mathrm{mg} / \mathrm{mL})$ was added and the plate again incubated for $30 \mathrm{~min}-$ utes. After 3 washes, streptavidin-PE was added with further incubation for 10 minutes. Beads were thoroughly mixed before reading on the Bio-Plex Suspension Array System. The data were analysed using BioPlexManager software with 5-parameter logistic (5PL) regression curves.

\subsection{Statistical analysis}

Skewed data were log transformed to achieve a more normal distribution of cytokine values. Differences in biological factor concentrations were compared between splenectomized and control groups by the nonparametric Kruskal-Wallis one-way analysis of variance (ANOVA). Dunn's method was used for all pairwise multiple comparisons to determine group differences. The ratios of median values of proinflammatory or angiostatic to anti-inflammatory or angiogenic cytokines were compared using ANOVA to assess alterations in the balance of Th1 and Th2 determinants between the groups. Box plots depicted factor concentrations (median, 25th and 75th percentiles) and values beyond the 10th and 90th percentiles were plotted as points. Statistical significance for each biological factor was set at a two-tailed $p<0.05$. SigmaPlot software version 10 (Systat Software Inc., San Jose, CA) was used to calculate statistics and plot graphs. Pair-wise comparisons were used to determine the level of significance of the differences between the plasma biological factor levels of the two groups (splenectomized group and control group). The plasma levels of 42 biological factors were grouped into proinflammatory, anti-inflammatory, and angiogenic/angiostatic biomarkers and cross analyzed.

\section{Results}

\subsection{Biomarker levels}

The proinflammatory and angiogenic/angiostatic levels demonstrated marginal changes but did not differ between the groups (data not shown). Among the antiinflammatory biomarkers, serum levels of IL-1a ( $p=$ $0.008)$ and CXCL10 ( $p=0.009)$ were significantly elevated in the splenectomized compared with the control group (Figs $1 \mathrm{~A}$ and B). When values were compared according to the indication for splenectomy, significant differences were confined to IL-1a $(p=0.028)$ for ASS versus controls (Figs $1 \mathrm{C}$ and D).

\subsection{Biomarker ratios in splenectomized and control individuals}

To determine the effect of splenectomy on Th1 and Th2 homeostasis, median ratios of plasma proinflammatory (IL-1 $\beta$, IL-2, IL-6, IL-8, IL-12, IL-15, IL-17, GM-CSF, IFN- $\gamma$, MCP-1, MIP- $1 \alpha$, MIP-1 $\beta$, RANTES, TNF), angiostatic (CXCL10, IL-12), antiinflammatory cytokines (IL-1ra, IL-4, IL-5, IL-6, IL9, IL-10, IL-13, G-CSF, Eotaxin) and angiogenic (IL-7, IL-8, bFGF-2, PDGF-bb, VEGF) were compared between splenectomized and control individuals. Biomarker median ratios of several cytokines were statistically higher when comparing splenectomized patients versus matched controls, in five biomarker ratios when comparing ASS versus controls and in two biomarkers in hypersplenic group versus controls (Table 1).

\section{Discussion}

The spleen is central to early pathology of patients with SS disease and either acute splenic sequestration or chronic hypersplenism occurred in $32 \%$ of newborns within in the first five years of life in the Jamaican Cohort Study [12] and were the second-leading causes of pediatric clinical complications in this study. Apart from possible contributions of $\mathrm{HbF}$ [15] and alphathalassemia, both factors which may influence sickling, is more complex since it allows splenomegaly to persist [7] and may contribute to the higher prevalence of splenomegaly among populations in eastern Saudi Arabia [11] and India [8]. However, the persistence of splenomegaly, consequent on a presumed inhibition of sickling, may also allow episodes of ASS to occur at a 


\section{IL-1 $\alpha$}

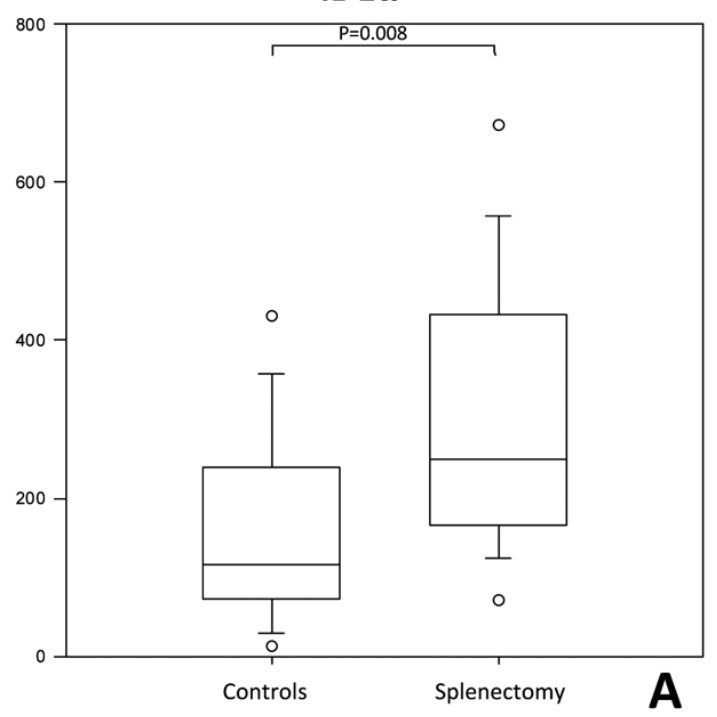

IL-1 $\alpha$

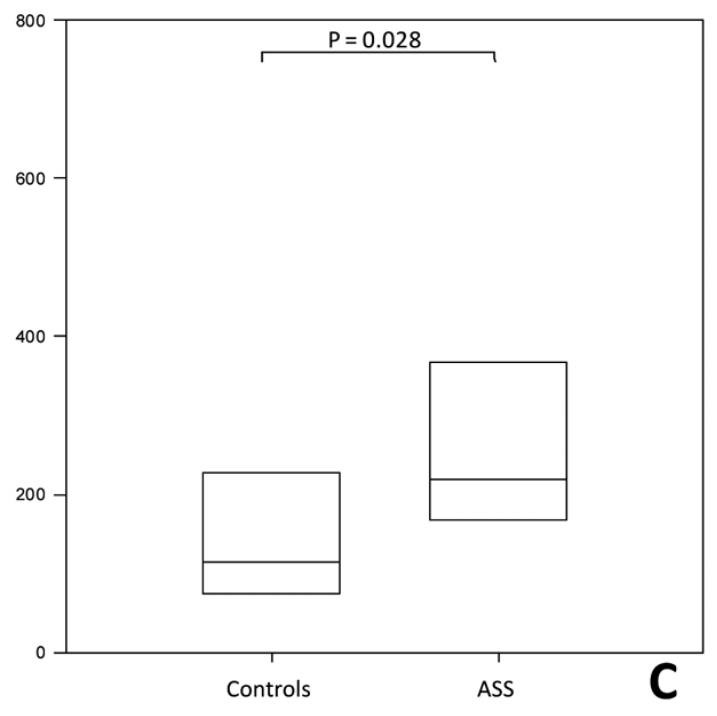

CXCL10

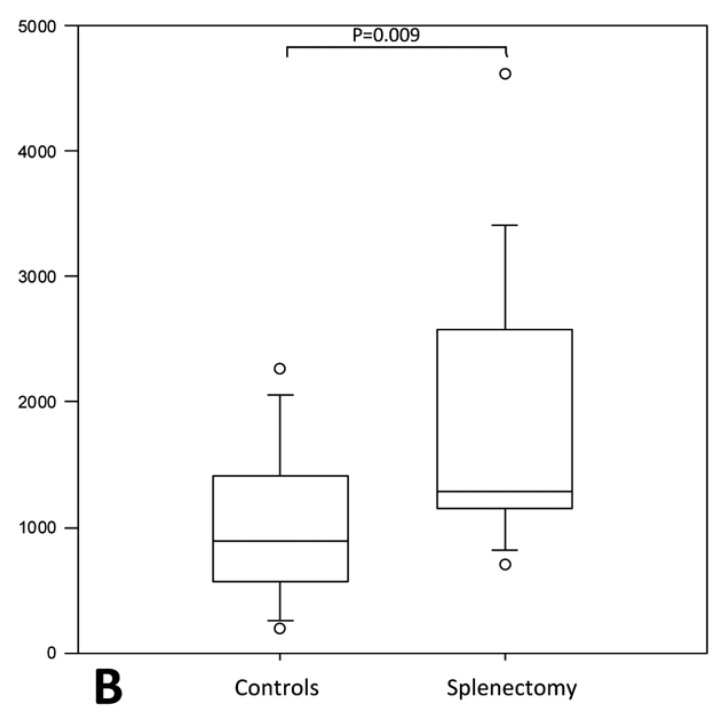

IL-1 $\alpha$

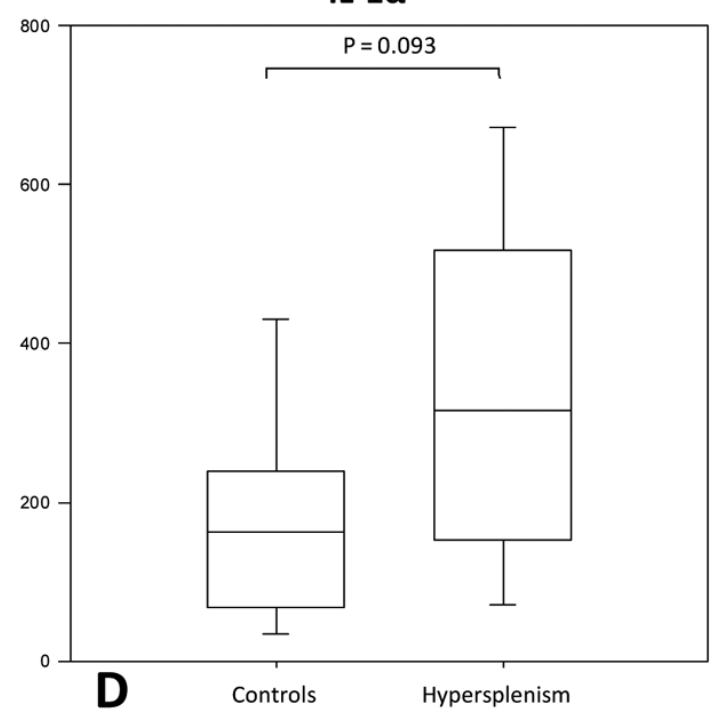

Fig. 1. A and B: IL-1 $\alpha$ and CXCL10 protein serum levels are significantly elevated in all splenectomized sub-groups vs control groups. C and D: IL- $1 \alpha$ protein serum levels are significantly elevated only in ASS (D) and not in hypersplenism (C) sub-group vs respective controls.

later age [2]. Apart from these effects of $\mathrm{HbF}$ and of alpha thalassemia, little is known of other genetic factors which might render patients prone to these splenic complications. Furthermore, a search for environmental factors was not supported by a seasonal pattern or clustering of events except for coincidence of attacks of ASS in identical twin studies [17]. The current study, although limited by sample size, used a powerful new technology to examine possible roles of cytokines, chemokines and/or growth factors in patients with a history of ASS or hypersplenism and matched controls available from the Jamaican Cohort Study. Studying these patients 25-30 years after their splenic complications also has the strength of identifying underlying genetic predispositions and avoids the hazards of events associated with active pathology.

The underlying hypothesis was that ASS and hypersplenism in SS disease may be influenced by host an- 
Table 1

Biomarker Ratios among Splenectomized and Normal Control Subjects (medians) and in those according to the Indication for Splenectomy

\begin{tabular}{lccccc}
\hline Biomarker median ratio & Controls & Splenectomy & $\begin{array}{c}\text { Overall } \\
\text { p value }\end{array}$ & $\begin{array}{c}\text { Hypersplenism } \\
\text { p value }\end{array}$ & $\begin{array}{c}\text { ASS } \\
\text { p value }\end{array}$ \\
\hline IL-1 $\alpha:$ IL-10 & 9.5 & 28.8 & 0.004 & 0.034 & 0.038 \\
IL-1 $\alpha:$ IL-1RA & 8.1 & 21.9 & 0.013 & 0.052 & 0.083 \\
IL-1 $\alpha$ :Eotaxin & 1.6 & 3.2 & 0.003 & 0.064 & 0.021 \\
IL-1 $\alpha:$ IL-6 & 34.4 & 114.6 & 0.005 & 0.161 & 0.005 \\
IL-1 $\alpha:$ G-CSF & 0.8 & 1.5 & 0.021 & 0.093 & 0.161 \\
IL-1 $\alpha:$ IL-9 & 41.5 & 109.5 & 0.029 & 0.329 & 0.121 \\
CXCL10:IL-7 & 220 & 498 & 0.038 & 0.024 & 0.955 \\
CXCL10:FGF-2 & 3.9 & 7.6 & 0.004 & 0.052 & 0.038 \\
CXCL10:PDGF-AA & 0.2 & 0.6 & 0.022 & 0.185 & 0.083 \\
CXCL10:PDGF-AA/AB & 0.06 & 0.1 & 0.009 & 0.077 & 0.105 \\
CXCL10:Flt-3 & 262 & 445 & 0.030 & 0.183 & 0.329 \\
CXCL10:EGF & 16.1 & 37.6 & 0.006 & 0.064 & 0.065 \\
IL-6:IL-5 & 1.1 & 0.3 & 0.012 & 0.152 & 0.041 \\
\hline
\end{tabular}

giopoietic and inflammatory mechanisms that are associated with polymorphisms in related biomarkers. The results indicate differences in specific cytokines between splenectomised subjects and controls, and also between those having splenectomy for ASS or hypersplenism, supporting the concept that patients developing ASS may have a specific and different phenotype from subjects with hypersplenism. The rationale for the differences between splenectomized patients is that they represent distinct phenotypic groups, possibly involving different polymorphisms that predispose them to these complications.

The biomarkers measured in this study consist of a diverse group of soluble proteins and peptides which act as regulators under both normal and pathological conditions to modulate the functional activities of individual cells and tissues. These proteins mediate interactions between cells directly and regulate processes taking place in the extracellular environment. They include lymphokines, interferons, colony stimulating factors and chemokines (cytokines with chemotactic activity). Additionally, cytokines and chemokines play a significant role in allergic reactions, cardiovascular disease, metabolic syndrome, sepsis and cancer. Therefore, our MILLIPLEX xMAP Human Cytokine/Chemokine panel enables us to focus on the curative potential of cytokines as well as the modulation of cytokine expression in a variety of therapeutic areas. Ultimately, confirmation studies on different populations will determine if the targeted genes are potential reliable biomarkers for use as diagnostic predictor(s). Confirmation of modifier genes will enable the discovery of pathways associated with each phenotype examined.

CXCL10 is a chemokine and ligand for the receptor CXCR3 and their binding results in pleiotropic effects, including stimulation of monocytes, natural killer and T-cell migration, and modulation of adhesion molecule expression. Polymorphisms in the CXCL10 gene have been described in idiopathic pulmonary fibrosis [9], the susceptibility to depression and antidepressant response [18] and disease progression of chronic HBV infection [3]. Finding single nucleotide polymorphisms (SNPs) associated with ASS or hypersplenism could aid in prediction of these complications and contribute to an understanding of their mechanism. Since IL$1 \alpha$ and CXCL10 were significantly different between the groups, the genes coding for these proteins need to be screened for polymorphisms such as have been described for the IL- $1 \alpha$ gene and its promoter for Alzheimer's disease [14] and rheumatoid arthritis [6].

The control group comprised SS patients in the same Cohort Study but without evidence of splenic complications. Compared with values from healthy subjects in the literature [1,19], levels of CXCL10 and IL-1a in these SS controls were higher consistent with the baseline inflammation inherent in SS disease. However, this control group was deemed more appropriate in assessing any changes of biomarkers in SS patients with splenic complications.

The current study is limited by small sample size but has several unique advantages. One of these is the close follow-up and documentation of all SS subjects in the Cohort rather than focusing on the severely affected cases as has happened in the past. This has been made possible by the protocols of the Cohort Study, by the geographical limitation of an island and the understanding and cooperation of Jamaican patients, so that no cases have been lost to the study after 38 years of follow-up. Furthermore the ability to study patients over 30 years after the occurrence of splenic patholo- 
gy more accurately reflects a genetic propensity rather than an acute response to the disordered hematology at the time of the complications. The choice of controls is also deemed especially appropriate as these have been followed in the same study from birth.

Future studies may be enhanced by new commercially available high throughput technologies to assess the translational potential of using such results toward improved patient care $[10,16]$. In the meantime, further exploration of these changes may contribute to an understanding of the mechanism and possible prediction of these splenic pathologies in the management of SS disease.

\section{Acknowledgments}

Authors thank the staff of the Sickle Cell Trust of Jamaica and of Morehouse School of Medicine. This work was supported by the National Institutes of Health grant numbers NIH-FIC (1T90-HG004151-01) for postdoctoral training in Genomics and Hemoglobinopathies, NIH/FIC/NINDS R21, NIH-RCMI (RR0330 62), and NIH-NHLBI \#R21HL092358-01. The funders had no role in study design, data collection and analysis, decision to publish, or preparation of the manuscript.

\section{References}

[1] A. Antonelli, P. Fallahi, M. Rotondi, S.M. Ferrari, M. Serio, and P. Miccoli, Serum levels of the interferon-gammainducible alpha chemokine CXCL10 in patients with active Graves' disease, and modulation by methimazole therapy and thyroidectomy, Br J Surg 93 (2006), 1226-1231.

[2] K. De Ceulaer and G.R. Serjeant, Acute splenic sequestration in Jamaican adults with homozygous sickle cell disease: a role of alpha thalassaemia, Br J Haematol 77 (1991), 563-564.

[3] G. Deng, G. Zhou, R. Zhang, Y. Zhai, W. Zhao, Z. Yan, C. Deng, X. Yuan, B. Xu, X. Dong, X. Zhang, X. Zhang, Z. Yao, Y. Shen, B. Qiang, Y. Wang and F. He, Regulatory polymorphisms in the promoter of CXCL10 gene and disease progression in male hepatitis B virus carriers, Gastroenterology 134 (2008), 716-726.

[4] A.M. Emond, R. Collis, D. Darvill, D.R. Higgs, G.H. Maude and G.R. Serjeant, Acute splenic sequestration in homozygous sickle cell disease: natural history and management, J Pediatr 107 (1985), 201-206.

[5] M.H. Gaston, J.I. Verter, G. Woods, C. Pegelow, J. Kelleher, G. Presbury, H. Zarkowsky, E. Vichinsky, R. Iyer, J.S. Lobel, et al. Prophylaxis with oral penicillin in children with sickle cell anemia. A randomized trial, N Engl J Med 314 (1986), 1593-1599.
[6] A. Havemose-Poulsen, L.K. Sorensen, K. Bendtzen and P. Holmstrup, Polymorphisms within the IL-1 gene cluster: effects on cytokine profiles in peripheral blood and whole blood cell cultures of patients with aggressive periodontitis, juvenile idiopathic arthritis, and rheumatoid arthritis, J Periodontol 78 (2007), 475-492.

[7] D.R. Higgs, B.E. Aldridge, J. Lamb, J.B. Clegg, D.J. Weatherall, R.J. Hayes, Y. Grandison, Y. Lowrie, K.P. Mason, B.E. Serjeant, and G.R. Serjeant, The interaction of alpha-thalassemia and homozygous sickle-cell disease, NEngl J Med 306 (1982), 1441-1446.

[8] B.C. Kar, R.K. Satapathy, A.E. Kulozik, M. Kulozik, S. Sirr, B.E. Serjeant and G.R. Serjeant, Sickle cell disease in Orissa State, India, Lancet 2 (1986), 1198-1201.

[9] L. Liu, H.P. Dai, B. Xiao, S. Zhang, C.J. Ban and P. Xin, [Association of ENA-78, IP-10 and VEGF gene polymorphism with idiopathic pulmonary fibrosis], Zhonghua Yi Xue Za Zhi 89 (2009), 2690-2694.

[10] R. Magi, A. Pfeufer, M. Nelis, A. Montpetit, A. Metspalu and M. Remm, Evaluating the performance of commercial wholegenome marker sets for capturing common genetic variation, BMC Genomics 8 (2007), 159.

[11] A.A. Mallouh and M.M. Salamah, Hypersplenism in homozygous sickle-cell disease in Saudi Arabia, Ann Trop Paediatr 5 (1985), 143-146.

[12] G.R. Serjeant and B.E. Serjeant, Management of sickle cell disease; lessons from the Jamaican Cohort Study, Blood Rev 7 (1993), 137-145.

[13] G.R. Serjeant, B.E. Serjeant, M. Forbes, R.J. Hayes, D.R. Higgs and H. Lehmann, Haemoglobin gene frequencies in the Jamaican population: a study in 100,000 newborns, $\mathrm{Br} \mathrm{J}$ Haematol 64 (1986), 253-262.

[14] A. Serretti, P. Olgiati, A. Politis, P. Malitas, D. Albani, S. Dusi, L. Polito, S. De Mauro, A. Zisaki, C. Piperi, I. Liappas, E. Stamouli, A. Mailis, A.R. Atti, M. Morri, M. Ujkaj, S. Batelli, G. Forloni, C.R. Soldatos, G.N. Papadimitriou, D. De Ronchi and A. Kalofoutis, Lack of association between interleukin1 alpha rs1800587 polymorphism and Alzheimer's disease in two Independent European samples, J Alzheimers Dis 16 (2009), 181-187.

[15] M.C. Stevens, R.J. Hayes, S. Vaidya and G.R. Serjeant, Fetal hemoglobin and clinical severity of homozygous sickle cell disease in early childhood, J Pediatr 98 (1981), 37-41.

[16] C. Timmann, J.A. Evans, I.R. Konig, A. Kleensang, F. Ruschendorf, J. Lenzen, J. Sievertsen, C. Becker, Y. Enuameh, K.O. Kwakye, E. Opoku, E.N. Browne, A. Ziegler, P. Nurnberg and R.D. Horstmann, Genome-wide linkage analysis of malaria infection intensity and mild disease, PLoS Genet 3 (2007), e48.

[17] M.W. Weatherall, D.R. Higgs, H. Weiss, D.J. Weatherall and G.R. Serjeant, Phenotype/genotype relationships in sickle cell disease: a pilot twin study, Clin Lab Haematol 27 (2005), 384-390.

[18] M.L. Wong, C. Dong, J. Maestre-Mesa and J. Licinio, Polymorphisms in inflammation-related genes are associated with susceptibility to major depression and antidepressant response, Mol Psychiatry 13 (2008), 800-812.

[19] Z.H. Yang, Q. Dai, L. Zhong, X. Zhang, Q.X. Guo and S.N. Li, Association of IL-1 polymorphisms and IL-1 serum levels with susceptibility to nasopharyngeal carcinoma, Mol Carcinog $\mathbf{5 0}$, $208-214$. 


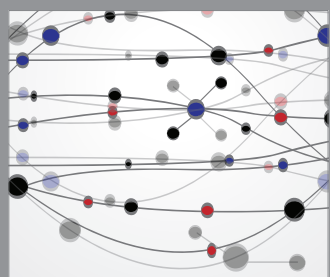

The Scientific World Journal
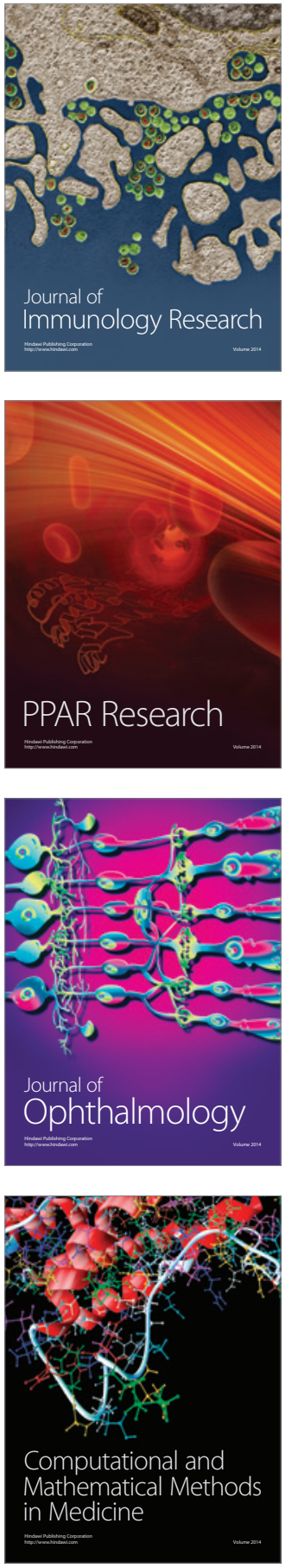

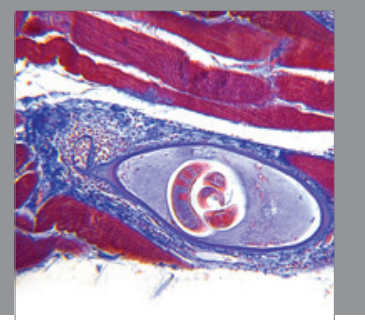

Gastroenterology

Research and Practice
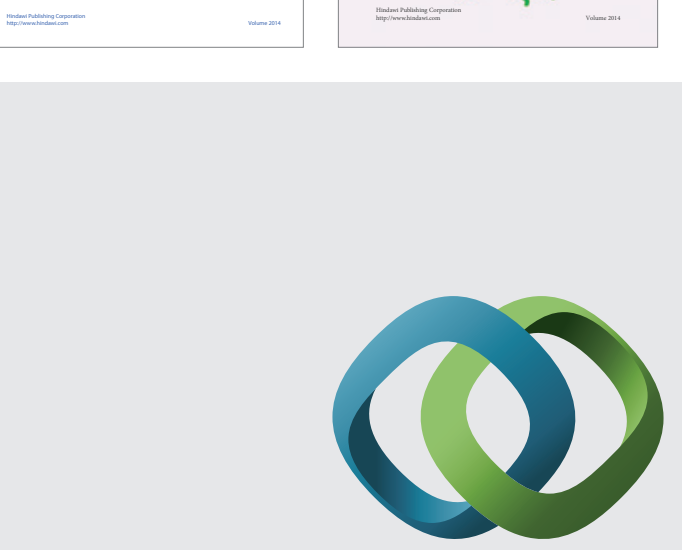

\section{Hindawi}

Submit your manuscripts at

http://www.hindawi.com
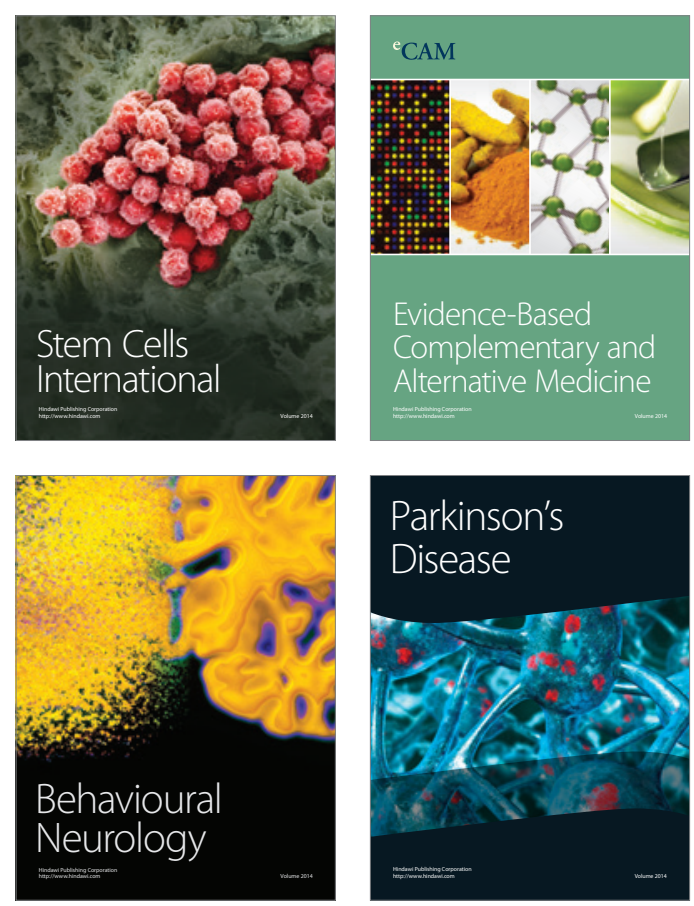

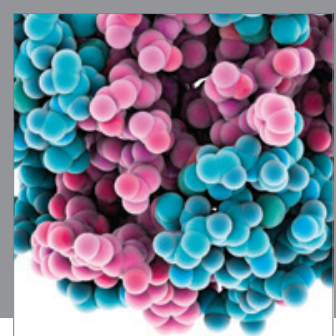

Journal of
Diabetes Research

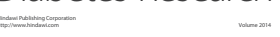

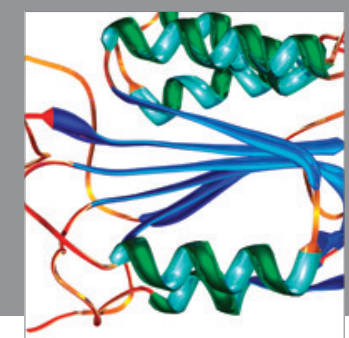

Disease Markers
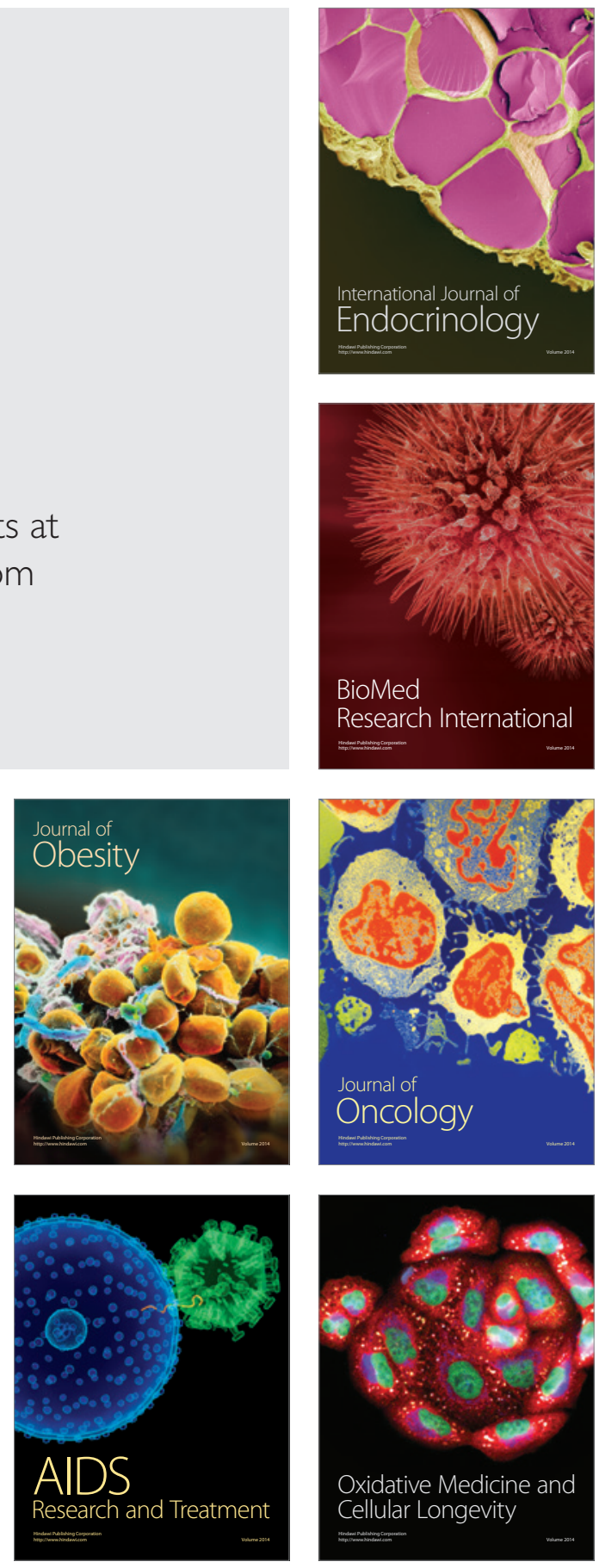